\title{
The Synthesis of New Isochromanylacetylarylhydrazones Designed as Probable Non-Addictive Analgesic Agents
}

\author{
Margareth Rôse L. Santos ${ }^{a}$, Eliezer J. Barreiro ${ }^{b^{*}}$, Raimundo Braz-Filho ${ }^{c}$, \\ and Ana Luisa P. Miranda ${ }^{b}$ \\ ${ }^{a}$ Curso de Pós-Graduação de Química Orgânica, Universidade Federal Rural do Rio \\ de Janeiro,23851-970 Seropédica, Rio de Janeiro - RJ, Brazil \\ ${ }^{b}$ Laboratório de Avaliação e Síntese de Substâncias Bioativas (LASSBio), Faculdade \\ de Farmácia, Universidade Federal do Rio de Janeiro, 21910-240 Rio de Janeiro - RJ, \\ Brazil,e-mail:eliezerb@unikey.com.br \\ ${ }^{c}$ Setor de Química de Produtos Naturais, LT-CCTA, Universidade Estadual do Norte \\ Fluminense, 28015-620 Campos, Rio de Janeiro - RJ, Brazil
}

Recieved: March 29, 1996; December 2, 1996

\begin{abstract}
A síntese e o perfil anti-inflamatório e analgésico dos novos agentes isocromanilacetilarilidrazônicos são descritos neste artigo. A rota sintética utilizada neste trabalho explora como passo chave para a construção de um anel heterocíclico de seis membros, a ciclização catalisada por um ácido de Lewis, que representa uma modificação da reação de Friedel-Crafts. Estes novos derivados (3) são obtidos em $c a$. de $85 \%$ de rendimento, a partir do safrol (4) utilizado como produto natural abundante, isolado do óleo de Sassafrás. A análise dos espectros de RMN destes novos derivados indica a natureza diastereoisomérica a nível da ligação $\mathrm{C}=\mathrm{N}$, na razão de 70:30, onde a maior contribuição é relativa ao isômero (E). Os resultados obtidos da avaliação farmacológica de (3) no teste do edema de pata de rato induzido por carragenina e no teste de contorções abdominais induzidas pelo ácido acético, indicam a natureza farmacofórica da unidade acilidrazona para a atividade analgésica observada nesta série. $O$ papel dos substituintes na sub-unidade arila na atividade farmacológica parece indicar que a presença de grupos hidrofóbicos podem elevar o perfil analgésico. Estes novos derivados isocromanilacetilarilidrazônicos (3) representam nova classe de agentes analgésicos não-convencionais.
\end{abstract}

The synthesis and pharmacological profile as analgesic and anti-inflammatory agents of new isochromanylacetylarylhydrazone derivatives (3) are described in this paper. The synthetic route used in this work to construct the heterocyclic six member ring explored a Lewis acid-catalyzed cyclization process as the key step, which represents a modified Friedel-Crafts reaction. These new derivatives (3) were obtained in $\mathrm{ca} .85 \%$ overall yields from the starting material safrole (4), an abundant natural product isolated from Sassafras oil. The NMR spectral analysis of these new derivatives indicated, at the $\mathrm{C}=\mathrm{N}$ double bond level, the diastereomeric nature in a 70:30 ratio, where the major compound is the $(E)$-isomer. The results obtained from the pharmacological evaluation of (3) using the carrageenan-induced rat paw edema test and the acetic acid solution-induced constrictions in mouse test, indicated the pharmacophoric nature of the acylarylhydrazone moiety to the analgesic activity observed in this series. The role of the aryl substituents in the bioactivity seems to indicate that the presence of hydrophobic groups may improve the analgesic profile. These new isochromanylacetylarylhydrazone derivatives (3) represent a new class of non-addictive analgesic agents.

Keywords: analgesic agents, isochromanylacetylarylhydrazones 


\section{Introduction}

Several cell mediators have been proposed to play a role in inflammatory processes and hyperalgesia, since these compounds caused a decrease in pain thresholds ${ }^{1}$. The prostaglandins PGs, in particular, have been suggested to play a major role in the pathogenesis of inflammation and in the production of inflammatory pain ${ }^{2-4}$. The recent disclosure of two distinct forms of cyclooxygenase (COX, prostaglandin-H synthase or PGHS $)^{5}$ indicated new directions for therapeutic approaches to inflammatory diseases ${ }^{6}$, confirming the role of icosanoids, produced by the COX enzyme which catalyzes the first two steps in the biosynthesis of PGs, producing the prostaglandin endoperoxide $\mathrm{G}_{2}$ and $\mathrm{H}_{2}$, precursors of all PGs. COX is the site of action of non-steroidal anti-inflammatory agents (NSAIS), and it is accepted that this is the basis of the anti-inflammatory action of these drugs ${ }^{7}$. The role of a second enzymatic pathway, namely 5 -lipooxygenase (5-LO) in inflammation is also well known ${ }^{8}$. From arachidonic acid (AA), this enzyme produces the leucotrienes (LTs) which present chemotactil and bronco-constriction properties, also playing an important role in some chronic inflammatory diseases?.

In an effort to develop new synthetic bioactive compounds, as part of a research program aiming at the synthesis and pharmacological evaluation of new non-addictive analgesic and anti-inflammatory agents ${ }^{10-17}$, we have previously described the synthesis and the anti-edematogenic properties of the isochromanyl acid derivatives $(1)^{16}$. These compounds showed significant anti-inflammatory and analgesic activity, reducing the edema in the carrageenan induced the rat paw test with a dose of $25 \mathrm{mg} / \mathrm{kg}$, and in reducing the number of constrictions in the acetic acid solution-induced test in mice with the same dose p.o. A second series of compounds, belonging to the acylhydrazone pyrazolic derivatives $(2)^{18}$ was also synthesized and pharmacologically evaluated for analgesic properties. These derivatives presented powerful activity in the mouse abdominal constrictions test induced by acetic acid ${ }^{11}$. The most potent compound of this series $\left(2 \mathrm{a}, \mathrm{W}=\mathrm{N}\left(\mathrm{CH}_{3}\right)_{2}\right)$ exhibited eleven times the analgesic activity of dipyrone in the same test at equimolar doses $\left(\mathrm{ED}_{50}=15.8 \mathrm{mg} / \mathrm{kg} ; P=\right.$ 0.05 ). These results led us to identify compound (3) as an attractive new target in the search for new anti-inflammatory and analgesic compounds. This new class of derivatives was designed as a structural hybrid of both (1) and (2), previously synthesized and elected as 'lead compounds', as shown in Scheme 1.

In this paper we describe the synthesis and pharmacological profile of these new isochromanylacetylaryl hydrazone derivatives (3), using safrole (4), an abundant natural Brazilian product available from Sassafras oil
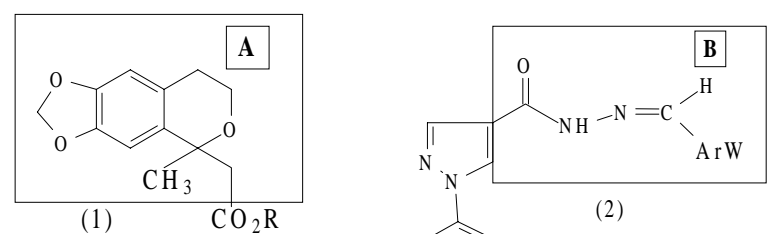

(2)
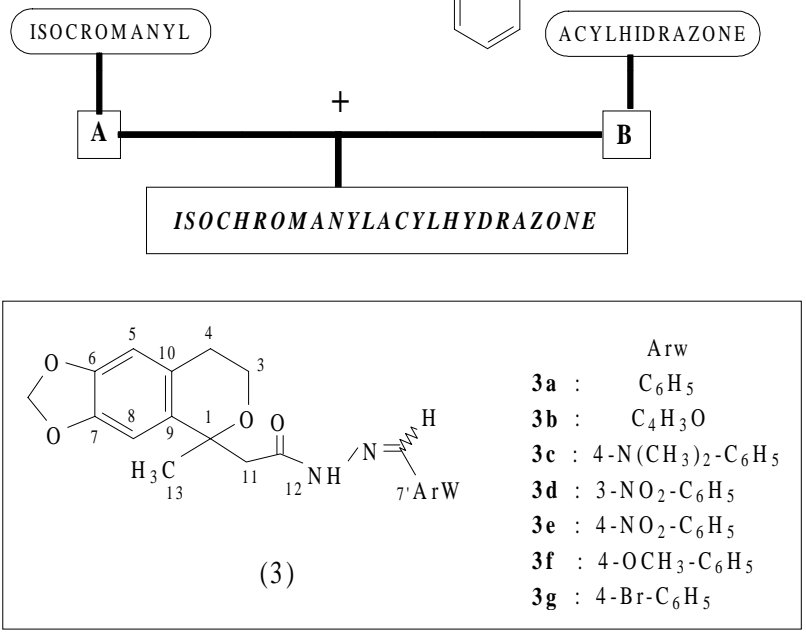

Scheme 1.

(Ocotea pretiosa Mer., Lauraceae), as the synthetic starting material $^{19}$.

\section{Experimental}

\section{Chemistry}

Melting points were determined with a hot-plate apparatus and were not corrected. Infrared (IR) spectra were obtained with a Perkin-Elmer 1600-FT spectrometer using potassium bromide pellets. Nuclear magnetic resonance $\left({ }^{1} \mathrm{H}-\mathrm{NMR}\right.$ and $\left.{ }^{13} \mathrm{C}-\mathrm{NMR}\right)$ spectra were recorded in deuterochloroform containing $c a$. 1\% TMS as an internal standard with $a$ Bruker AC-200 FT at $200 \mathrm{MHz}\left({ }^{1} \mathrm{H}\right)$ and $50.3 \mathrm{MHz}\left({ }^{13} \mathrm{C}\right)$. Chemical shifts are expressed in ppm $(\delta)$ relative to TMS $(=0 \mathrm{ppm})$. Splitting patterns are as follows: $\mathrm{s}$, singlet; d, doublet; t, triplet; q, quartet; m, multiplet; br, broad. The mass spectra were obtained with a GC/VG Micromass 12 at $70 \mathrm{eV}$. The progress of all reactions was monitored by thin layer chromatography (TLC), using precoated Merck silica gel $60 \mathrm{~F}_{254}$ aluminum plates; detection was done with UV and $\mathrm{I}_{2}$. For column chromatography, Merck silica gel (70-230 mesh) was used. Solvents used in the reactions were generally redistilled prior to use and stored over 3A molecular sieves.

Ethyl ester of [1-(1-methyl-6,7-methylenedioxy)-isochromanyl]- acetic acid $(5)^{16}$

Ethyl acetoacetate $(1.80 \mathrm{~g} ; 10.98 \mathrm{mmol})$ was added to a solution of homopiperonilic alcohol (8) (1.62 g, 12.6 mmol) in dry benzene $(30 \mathrm{~mL})$. A catalytic amount of p-toluenesulfonic acid ( $c a .0 .2 \mathrm{~g}$ ) was added to the flask 
equipped with a Dean-Stark trap, and the reaction mixture was refluxed for $12 \mathrm{~h}$. The solvent was removed under reduced pressure, affording a pale yellow oil which was dissolved in ethyl ether (ca. $30 \mathrm{~mL})$. The oil was washed with a $30 \%$ aqueous sodium hydroxide solution. The organic layer (negative $\mathrm{FeCl}_{3}$ test) was dried over sodium sulfate, filtered, evaporated, and then chromatographed on a silica gel column (n-hexane:ethyl acetate, 9:1) to give the desired product (5) in a 75\% yield. Its IR and $60 \mathrm{MHz}{ }^{1} \mathrm{H}$ NMR have already been described ${ }^{16}$. New data are reported.

M.S. (70 eV): 278 (7\%); 191 (100\%); 149 (9\%); 110 (31\%).

${ }^{1} \mathrm{H}-\mathrm{NMR}(200 \mathrm{MHz}) \mathrm{CDCl}_{3} / \mathrm{TMS} \delta: 6.56(\mathrm{~s}, 2 \mathrm{H}, \mathrm{H}-5$ and $\mathrm{H}-8) ; 5.9\left(\mathrm{~s}, 2 \mathrm{H}, \mathrm{OCH}_{2} \mathrm{O}\right) ; 4.10\left(\mathrm{q}, 2 \mathrm{H}, \mathrm{OC}_{2} \mathrm{CH}_{3}\right) ; 3.91$ $(\mathrm{m}, 2 \mathrm{H}, \mathrm{H}-3) ; 2.90(\mathrm{~d}, \mathrm{~J}=13.5 ; 1 \mathrm{H}, \mathrm{H}-11) ; 2.73(\mathrm{~d}, \mathrm{~J}=$ 13.5;1H, H-11); 2.71 (m,2H,H-4); 1.27 (s,3H, H-13); 1.20 $\left(\mathrm{t}, 3 \mathrm{H}, \mathrm{OCH}_{2} \mathrm{CH}_{3}\right)$.

${ }^{13} \mathrm{C}-\mathrm{NMR}(50.3 \mathrm{MHz}) \mathrm{CDCl}_{3} / \mathrm{TMS} \delta: 169.98(\mathrm{C}=\mathrm{O})$; 145.94 (C-6); 133.78 (C-7); 125.45 (C-9); 115.88 (C-10); 108.28 (C-5); $105.18(\mathrm{C}-8) ; 100.63\left(\mathrm{OCH}_{2} \mathrm{O}\right) ; 75.20(\mathrm{C}-1)$; $60.11\left(\mathrm{OCH}_{2} \mathrm{CH}_{3}\right) ; 60.01(\mathrm{C}-3) 46.40(\mathrm{C}-11) ; 29.60(\mathrm{C}-4)$; $27.00(\mathrm{C}-13) ; 12.00\left(\mathrm{OCH}_{2} \mathrm{CH}_{3}\right)$.

\section{[1-(1-Methyl-6,7-methylenedioxy)-isochromanyl]-acetyl hydrazide (6).}

An ethanolic solution of ester (5) (1.30 g; $4.65 \mathrm{mmol})$ in $25 \mathrm{~mL}$ of ethanol was heated under stirring until complete dissolution. Then $4.7 \mathrm{~mL}$ of $85 \%$ hydrazine hydrate was added, and the reaction mixture was stirred at reflux for $3 \mathrm{~h}$. At the end of the reaction (as indicated by TLC analysis) the product (6) was isolated by concentration of the reaction mixture under reduced pressure. Cold water was added and the resulting product (6) was filtered to give $1.10 \mathrm{~g}(85 \%)$ of a white solid.

m.p.:154- $155^{\circ} \mathrm{C}$.

I.R. $(\mathrm{KBr})$ v: $3360(\mathrm{NH}) ; 1670(\mathrm{C}=\mathrm{O}) \mathrm{cm}^{-1}$.

M.S. (70 eV) m/z,: 264 (13\%); 192 (24\%); 191 (100\%) $115(7 \%) 91(6 \%) ; 77$ ( $5 \%)$.

${ }^{1} \mathrm{H}-\mathrm{NMR}(200 \mathrm{MHz}) \mathrm{CDCl}_{3} / \mathrm{TMS} \delta: 7.76$ (br. $\mathrm{s}, 1 \mathrm{H}, \mathrm{NH}) ; 6.53$ (s,1H, H-8); 6.50 (s,1H,H-5); 5.87 $\left(\mathrm{m}, 2 \mathrm{H}, \mathrm{OCH}_{2} \mathrm{O}\right) ; 3.85$ (m,2H,H-3); 3.76 (br. s, $\left.2 \mathrm{H}, \mathrm{NH}_{2}\right)$; 2.60 (m,2H,H-4); 2.73 (m,1H,H-11); 2.68 (m ,1H, H-11); 1.48 (s,3H,H-13).

${ }^{13} \mathrm{C}-\mathrm{NMR}(50.3 \mathrm{MHz}) \mathrm{CDCl}_{3} / \mathrm{TMS} \delta: 170.70(\mathrm{C}=\mathrm{O})$; 146.16 (C-6,7); 132.61 (C-9); 125.97 (C-10); 108.21 (C-5); 105.26 (C-8); $100.73\left(\mathrm{OCH}_{2} \mathrm{O}\right) ; 75.42(\mathrm{C}-1) ; 59.47$ (C-3); 47.38 (C-11); 29.00 (C-4); 27.09 (C-13).

The general procedure for preparing [1-(1-Methyl-6,7methylenedioxy)-isochromanyl]-acetylarylhydrazones $(3 a-3 g)$

Benzaldehyde or its derivative $(0.57 \mathrm{mmol})$ was added to a solution of hydrazine derivative $(6)(0.15 \mathrm{~g} ; 0.57 \mathrm{mmol})$ in absolute ethanol, in the presence of two drops of hydrochloric acid as a catalyst. The end of the reaction was detected by TLC and the new hydrazones (3a-3g) were isolated by the concentration of the reaction mixture under reduced pressure and the addition of cold water to furnish the desired product as a colored precipitate in an $80 \%$ yield (Table 1). The E and $\mathrm{Z}$ isomers could not be separated, but their ${ }^{1} \mathrm{H}$ and ${ }^{13} \mathrm{C}$ assignments could be made from their higher field $2 \mathrm{D}$ spectra $\left({ }^{1} \mathrm{Hx}^{1} \mathrm{H}-\mathrm{COSY},{ }^{1} \mathrm{Hx}^{13} \mathrm{C}\right.$ COSY,COLOC).

[1-(1-Methyl-6,7-methylenedioxy)-isochromanyl]-acetyl arylhydrazone of benzaldeyde $(3 a-(E),(Z))$

m.p.: $78-80{ }^{\circ} \mathrm{C}$

I.R:. (KBr) v: 3430, $3200(\mathrm{NH}) ; 1664(\mathrm{C}=\mathrm{O}) \mathrm{cm}^{-1}$.

M.S. (70 eV) m/z: 352 (3\%); 208 (8\%); 192 (14\%); 191 $(100 \%) ; 170(11 \%)$.

(E) Isomer

${ }^{1} \mathrm{H}-\mathrm{NMR}(200 \mathrm{MHz}) \mathrm{CDCl}_{3} / \mathrm{TMS}(\delta=\mathrm{ppm}): 9.83$ (s,1H,NH); 8.00 (s,1H,H-7'); 7.70 (m,2H,H-2'6'); 7.4 (m,3H,H-3' 5', 4'); 6.59 (s,1H,H-8); 6.50 (s,1H,H-5); 5.9 $\left(\mathrm{s}, 2 \mathrm{H}, \mathrm{OCH}_{2} \mathrm{O}\right) ; 4.1(\mathrm{~m}, 2 \mathrm{H}-3) ; 2.9(\mathrm{~m}, 2 \mathrm{H}-11) ; 2.8(\mathrm{~m}, 2 \mathrm{H}-$ 4); 1,56 (s,3H-13).

${ }^{13} \mathrm{C}-\mathrm{NMR}(50.3 \mathrm{MHz}) \mathrm{CDCl}_{3} / \mathrm{TMS}(\delta=\mathrm{ppm}): 166.45$ (C=O); 147.10 (C-7'); 146.37 (C-7); 146.25 (C-6); 133.48 (C-1'); 132.34 (C-9); 130.12 (C-4'); 128.39 (C-3'5'); 127.46 (C-2'6'); 126.48 (C-10); 100.80 (OCH2O);75.97 (C-1);59.72 (C-3);48.37 (C-11) ;29.18 (C-4);27.20 (C-13).

Table 1. The physical properties of the new isochromanylacetyl hydrazone derivatives (3).

\begin{tabular}{lccccc}
\hline Compounds & $\mathrm{ArW}$ & Molecular Formula & Yield (\%) & Molecular weight & m.p. $\left({ }^{\circ} \mathrm{C}\right)$ \\
\hline $3 \mathrm{a}$ & $\mathrm{C}_{6} \mathrm{H}_{5}$ & $\mathrm{C}_{20} \mathrm{H}_{20} \mathrm{O}_{4} \mathrm{~N}_{2}$ & 80 & 352 & $78-80$ \\
$3 \mathrm{~b}$ & $\mathrm{C}_{4} \mathrm{H}_{3} \mathrm{O}$ & $\mathrm{C}_{18} \mathrm{H}_{18} \mathrm{O}_{5} \mathrm{~N}_{2}$ & 88 & 342 & $88-91$ \\
$3 \mathrm{c}$ & $4-\mathrm{N}\left(\mathrm{CH}_{3}\right)_{2}-\mathrm{C}_{6} \mathrm{H}_{4}$ & $\mathrm{C}_{22} \mathrm{H}_{25} \mathrm{O}_{4} \mathrm{~N}_{3}$ & 94 & 395 & $93-94$ \\
$3 \mathrm{~d}$ & $3-\mathrm{NO}_{2}-\mathrm{C}_{6} \mathrm{H}_{4}$ & $\mathrm{C}_{20} \mathrm{H}_{19} \mathrm{O}_{6} \mathrm{~N}_{3}$ & 75 & 397 & $183-184$ \\
$3 \mathrm{e}$ & $4-\mathrm{NO}_{2}-\mathrm{C}_{6} \mathrm{H}_{4}$ & $\mathrm{C}_{20} \mathrm{H}_{19} \mathrm{O}_{6} \mathrm{~N}_{3}$ & 80 & 397 & $226-228$ \\
$3 \mathrm{f}$ & $4-\mathrm{OMe}_{-} \mathrm{C}_{6} \mathrm{H}_{4}$ & $\mathrm{C}_{21} \mathrm{H}_{22} \mathrm{O}_{5} \mathrm{~N}_{2}$ & 85 & 382 & $168-169$ \\
$3 \mathrm{~g}$ & $4-\mathrm{Br}_{-} \mathrm{C}_{6} \mathrm{H}_{4}$ & $\mathrm{C}_{20} \mathrm{H}_{19} \mathrm{O}_{4} \mathrm{~N}_{2} \mathrm{Br}$ & 80 & 440 & $178-179$ \\
\hline
\end{tabular}


(Z) Isomer

${ }^{1} \mathrm{H}-\mathrm{NMR}(200 \mathrm{MHz}) \mathrm{CDCl}_{3} / \mathrm{TMS}(\boldsymbol{\delta}=\mathrm{ppm}): 9.18$ (s,1H,NH); 7.70 (s,1H,H-7'); 7.61 (m,2H,H-2' $\left.6^{\prime}\right) ; 7.4$ (m,3H,H-3' 5',4'); 6.66 (s,1H,H-8); 6.52 (s,1H,H-5); 5.9 $\left(\mathrm{s}, 2 \mathrm{H}, \mathrm{OCH}_{2} \mathrm{O}\right) ; 4.0(\mathrm{~m}, 2 \mathrm{H}-3) ; 3.46(\mathrm{~d}, \mathrm{~J}=14,1 \mathrm{H}-11) ; 3.10$ $(\mathrm{d}, \mathrm{J}=14,1 \mathrm{H}-11) ; 2.75$ (m,2H-4);1.63 (s,3H-13).

${ }^{13} \mathrm{C}-\mathrm{NMR}(50.3 \mathrm{MHz}) \mathrm{CDCl}_{3} / \mathrm{TMS}(\boldsymbol{\delta}=\mathrm{ppm}): 172.87$ $(\mathrm{C}=\mathrm{O}) ; 143.75$ (C-7'); 145.89 (C-7); 145.77 (C-6); 134.58 (C-9); 133.85 (C-1'); 129.76 (C-4'); 128.54 (C-3'5'); 126.91 (C-2'6'); 125.79 (C-10); 100.60 (OCH2O); 75.88 (C-1); 59.86 (C-3); 42.49 (C-11); 29.24 (C-4); 28.36 (C$13)$.

[1-(1-Methyl-6,7-methylenedioxy)-isochromanyl]-acetyl arylhydrazone of 2-furaldeyde (3b-(E),(Z))

m.p.: $88-91{ }^{\circ} \mathrm{C}$

I.R.(KBr) v: $3450,3215(\mathrm{NH}) ; 1670(\mathrm{C}=\mathrm{O}) \mathrm{cm}^{-1}$.

(E) Isomer

${ }^{1} \mathrm{H}-\mathrm{NMR}(200 \mathrm{MHz}) \mathrm{CDCl}_{3} / \mathrm{TMS}(\delta=\mathrm{ppm}): 9.79$ $(\mathrm{s}, 1 \mathrm{H}, \mathrm{NH}) ; 8.21$ (s,1H,H-5'); 7.46 (s,2H,H-4); 7.24 (s,2H,H-2'6'); 6.67 (m,2H,H-3' 5'); 6.57 (s,1H,H-8); 6.49 $(\mathrm{s}, 1 \mathrm{H}, \mathrm{H}-5) ; 5.88\left(\mathrm{~s}, 2 \mathrm{H}, \mathrm{OCH}_{2} \mathrm{O}\right) ; 3.8(\mathrm{~m}, 2 \mathrm{H}-3) ; 2.65$ $(\mathrm{m}, 2 \mathrm{H}-4) ; 2.87(\mathrm{~d}, \mathrm{~J}=14,2 \mathrm{H}-11) ; 2.83(\mathrm{~d}, \mathrm{~J}=14,2 \mathrm{H}-11)$; 1.54 (s,3H-13).

${ }^{13} \mathrm{C}-\mathrm{NMR}(50.3 \mathrm{MHz}) \mathrm{CDCl}_{3} / \mathrm{TMS}(\delta=\mathrm{ppm}): 166.60$ $(\mathrm{C}=\mathrm{O}) ; 149.06\left(\mathrm{C}^{\prime} 1^{\prime}\right) ; 146.19$ (C-7); 146.10 (C-6); 144.17 (C-4'); 137.31 (C-5'); 132.36 (C-9); 133.38 (C-2'); 125.82 (C-10); 112.87 (C-3'); 108.04 (C-5); 105.29 (C-8); 100.68 $\left(\mathrm{OCH}_{2} \mathrm{O}\right) ; 75.79$ (C-1); 59.57 (C-3); 48.54 (C-11); 29.03 (C-4); 27.15 (C-13).

(Z) Isomer

${ }^{1} \mathrm{H}-\mathrm{NMR}(200 \mathrm{MHz}) \mathrm{CDCl}_{3} / \mathrm{TMS}(\delta=\mathrm{ppm}): 9.40$ $\left.(\mathrm{s}, 1 \mathrm{H}, \mathrm{NH}) ; 7.60\left(\mathrm{~s}, 1 \mathrm{H}, \mathrm{H}^{-}\right)^{\prime}\right) ; 6.64(\mathrm{~s}, 1 \mathrm{H}, \mathrm{H}-8) ; 6.44$ $(\mathrm{s}, 1 \mathrm{H}, \mathrm{H}-5) ; 5.88\left(\mathrm{~s}, 2 \mathrm{H}, \mathrm{OCH}_{2} \mathrm{O}\right) ; 4.0(\mathrm{~m}, 2 \mathrm{H}-3) ; 3.45(\mathrm{~d}, \mathrm{~J}=$ 14, 1H,H-11); 3.03 (d,J = 14, 1H,H-11); 2.90 (m, 2H-4); 1.60 (s, 3H-13).

${ }^{13} \mathrm{C}-\mathrm{NMR}(50.3 \mathrm{MHz}) \mathrm{CDCl}_{3} / \mathrm{TMS}(\boldsymbol{\delta}=\mathrm{ppm}): 172.78$ $(\mathrm{C}=\mathrm{O}) ; 149.19$ (C-1'); 145.77 (C-7); 145.70 (C-6); 144.00 (C-4'); 134.54 (C-9); 137.39 (C-5'); 111.63 (C-5); 111.63 (C-8); 100.50 (OCH2O); 75.79 (C-1); 59.71 (C-3); 43.31 (C-11); 28.75 (C-4); 28.33 (C-13).

[1-(1-Methyl-6,7-methilenedioxy)-isochromanyl]-acetyl arylhydrazone of 4-dimethylaminobenzaldeyde (3c$(E),(Z))$

m.p.: $93-94{ }^{\circ} \mathrm{C}$

I.R. (KBr) v: 3450, $3201(\mathrm{NH}) ; 1655(\mathrm{C}=\mathrm{O}) \mathrm{cm}^{-1}$.

M.S.(70 eV) m/z: 395 (4\%); 191 (100\%); 149 (50\%); 148 (65\%); 135 (18\%); 77 (18\%).

(E) Isomer

${ }^{1} \mathrm{H}-\mathrm{NMR}(200 \mathrm{MHz}) \mathrm{CDCl}_{3} / \mathrm{TMS}(\delta=\mathrm{ppm}): 9.64$ (s,1H,NH); 7.83 (s,1H,H-7'); 7.57 (d,J = 9,2H,H-2'6'); $6.62\left(\mathrm{~d}, \mathrm{~J}=9,2 \mathrm{H}, \mathrm{H}-3^{\prime} 5^{\prime}\right) ; 6.59$ (s,1H,H-8); 6.49 (s,1H,H-5); $5.9\left(\mathrm{~m}, 2 \mathrm{H}, \mathrm{OCH}_{2} \mathrm{O}\right) ; 4.1(\mathrm{~m}, 2 \mathrm{H}-3) ; 2.9(\mathrm{~m}, 2 \mathrm{H}-4) ; 2.98$ $\left(\mathrm{s}, \mathrm{N}\left(\mathrm{CH}_{3}\right)_{2}\right) ; 2.9(\mathrm{~d}, \mathrm{~J}=14,2 \mathrm{H}-11) ; 2.8(\mathrm{~d}, \mathrm{~J}=14,2 \mathrm{H}-11)$; 1.55 (s, 3H-13).

${ }^{13} \mathrm{C}-\mathrm{NMR}(50.3 \mathrm{MHz}) \mathrm{CDCl}_{3} / \mathrm{TMS}(\delta=\mathrm{ppm}) .: 165.84$ $(\mathrm{C}=\mathrm{O}) ; 151.44$ (C-4'); 147.90 (C-7'); 146.19 (C-6); 146.17 (C-7); 132.52 (C-9); 128.89 (C-2'6'), 125.76 (C-10); 120.96(C-1'); 111.32 (C-3'5'); 108.14(C-5); $105.46(\mathrm{C}-8)$; $100.65\left(\mathrm{OCH}_{2} \mathrm{O}\right) ; 75.82(\mathrm{C}-1) ; 59.56(\mathrm{C}-3) ; 48.07$ (C-11);

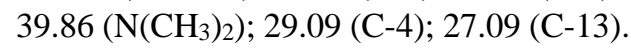

(Z) Isomer

${ }^{1} \mathrm{H}-\mathrm{NMR}(200 \mathrm{MHz}) \mathrm{CDCl}_{3} / \mathrm{TMS}(\delta=\mathrm{ppm}): 8.90$ $(\mathrm{s}, \mathrm{NH}) ; 7.58$ (s,H-7'); 7.47 (d,J = 9,H-2'6'); 6.66 (d,J = 9,H-3'5'); 6.61 (s,H-8); 6.52 (s,H-5); 5.9 m, $\mathrm{OCH}_{2} \mathrm{O}$ ); 4.1 (m, 2H-3), $3.50(\mathrm{~d}, \mathrm{~J}=15,2 \mathrm{H}-11) ; 3.06(\mathrm{~d}, \mathrm{~J}=15,2 \mathrm{H}-11)$; $2.99\left(\mathrm{~s}, \mathrm{~N}\left(\mathrm{CH}_{3}\right)_{2}\right) ; 2.9$ (m, 2H-4); 1.62 (s, 3H-13).

${ }^{13} \mathrm{C}-\mathrm{NMR}(50.3 \mathrm{MHz}) \mathrm{CDCl}_{3} / \mathrm{TMS}(\boldsymbol{\delta}=\mathrm{ppm}): 171.97$ $(\mathrm{C}=\mathrm{O})$; $151.20\left(\mathrm{C}^{\prime} 4^{\prime}\right) ; 146.00(\mathrm{C}-6,7) ; 144.48\left(\mathrm{C}^{\prime} 7^{\prime}\right)$; 134.76 (C-9): 128.16 (C-2'6'); 126.33 (C-10); 121.54 (C1'); 111.55 (C3'5'); 108.14 (C-5); 105.32 (C-8); 100.49 (OCH2O); 75.82 (C-1); 59.72 (C-3); 42.29 (C-11); 39.93 (N(CH3)2); 29.15 (C-4); 28.50 (C-13).

[1-(1-Methyl-6,7-methylenedioxy)-isochromanyl]-acetyl arylhydrazone of m-nitrobenzaldehyde $(3 d-(E),(Z))$

m.p.: $183-184{ }^{\circ} \mathrm{C}$

I.R.. (KBr) v: 3393, $3193(\mathrm{NH}) ; 1665(\mathrm{C}=\mathrm{O}) \mathrm{cm}^{-1}$.

M.E.(70 eV): 397 (3\%); 217 (6\%) ; 191 (100\%); 166 (17\%); 135 (63\%);77 (15\%).

(E) Isomer

${ }^{1} \mathrm{H}-\mathrm{NMR}(200 \mathrm{MHz}) \mathrm{CDCl}_{3} / \mathrm{TMS}(\delta=\mathrm{ppm}): 10.02$ (s,NH); 8.43 (H-7'); 8.20 (H-2') 8.11 (H-4'); 7.90 (H-6'); 6.58 (s,H-8); 6.49 (s,H-5); $5.87\left(\mathrm{OCH}_{2} \mathrm{O}\right) ; 3.98$ (m, 2H-3); 2.89 (2H-11); 2.87 (2H-11); 2.84 (m, 2H-4); 1.55 (s, 3H13).

${ }^{13} \mathrm{C}-\mathrm{NMR}(50.3 \mathrm{MHz}) \mathrm{CDCl}_{3} / \mathrm{TMS}(\delta=\mathrm{ppm}): 166.91$ $(\mathrm{C}=\mathrm{O}) ; 148.35$ (C-3'); 146.67 (C-6,7); 144.88 (C-7'); 135.67 (C-1'); 132.58 (C-6'); 132.33 (C-9); 129.56 (C-5'); 124.38 (C-4'); 122.25 (C-2'); 108.43 (C-5); 105.47 (C-8); $100.96\left(\mathrm{OCH}_{2} \mathrm{O}\right) ; 76.10(\mathrm{C}-1) ; 59.97(\mathrm{C}-3) ; 48.96(\mathrm{C}-11)$; 29.37 (C-4); 27.36 (C-13).

(Z) Isomer

${ }^{1} \mathrm{H}-\mathrm{NMR}(200 \mathrm{MHz}) \mathrm{CDCl}_{3} / \mathrm{TMS}(\delta=\mathrm{ppm}) .: 9.96$ (s,NH); 8.43 (H-7'); 7.81 (H-6'); 6.64 ( H-8); 6.57 ( H-5); $6.16\left(\mathrm{H}-2^{\prime}\right) ; 5.82\left(\mathrm{OCH}_{2} \mathrm{O}\right) ; 4.06(\mathrm{~m}, 2 \mathrm{H}-3) 3.41(2 \mathrm{H}-11)$; 3.18 (2H-11); 2.84 (m, 2H-4).

${ }^{13} \mathrm{C}-\mathrm{NMR}(50.3 \mathrm{MHz}) \mathrm{CDCl}_{3} / \mathrm{TMS}(\boldsymbol{\delta}=\mathrm{ppm}):: 172.97$ $(\mathrm{C}=\mathrm{O})$; 148.40 (C-3'); 146.04 (C-6,7); 136.00 (C-7'); 135.76 (C-1'); 132.33 (C-9); 129.56 (C-5'); 126.00 (C-10); 124.11 (C-4'); 121.58 (C-2'); 108.43 (C-5); 105.47 (C-8); $100.75\left(\mathrm{OCH}_{2} \mathrm{O}\right) ; 76.10(\mathrm{C}-1) ; 59.97(\mathrm{C}-3) ; 43.14$ (C-11); 29.37 (C-4); 28.52 (C-13). 
[1-(1-Methyl-6,7-methylenedioxy)-isochromanyl] acetylarylhydrazone of p-nitrobenzaldehyde $(3 e-(E),(Z))$

m.p.: $226228^{\circ} \mathrm{C}$

I.R. (KBr ) v: 3413, $3170(\mathrm{NH}) ; 1665(\mathrm{C}=\mathrm{O}) \mathrm{cm}^{-1}$.

M.E.: 397 (6\%); 298 (22\%); 191 (100\%); 176 (32\%); $149(21 \%) ; 57(22 \%)$.

(E) Isomer

${ }^{1} \mathrm{H}-\mathrm{NMR}(200 \mathrm{MHz}) \mathrm{CDCl}_{3} / \mathrm{TMS}(\boldsymbol{\delta}=\mathrm{ppm}) .: 9.9(\mathrm{~s}$, $\mathrm{NH}) ; 8.70$ (s, H-7'); 8.23 (H-3' 5'); 7.86 (m, H-2'6'); 6.52 (H-5); $6.50(\mathrm{H}-8) ; 5.89\left(\mathrm{OCH}_{2} \mathrm{O}\right) ; 4.05(2 \mathrm{H}-3) ; 2.89(2 \mathrm{H}-$ 11); 2.67 (2H-4); 1.58 (3H-13).

${ }^{13} \mathrm{C}-\mathrm{NMR}(50.3 \mathrm{MHz}) \mathrm{CDCl}_{3} / \mathrm{TMS}(\boldsymbol{\delta}=\mathrm{ppm}) .: 145.38$ (C-6); 145.20 (C-7); 125.79 (C-10); 107.55 (C-5); 105.14 (C-8); $100.15\left(\mathrm{OCH}_{2} \mathrm{O}\right) ; 75.07$ (C-1); 59.87 (C-3); 46.78 (C-11); 28.57 (C-4); 27.90 (C-13).

(Z) Isomer

${ }^{1} \mathrm{H}-\mathrm{NMR}(200 \mathrm{MHz}) \mathrm{CDCl}_{3} / \mathrm{TMS}(\boldsymbol{\delta}=\mathrm{ppm}) .: 9.90$ (NH); 8.33 (H-7'); 8.19 (H-3'5'); 7.85 (H-2'6'); 6.52 (H5); $6.60(\mathrm{H}-8) ; 5.89\left(\mathrm{OCH}_{2} \mathrm{O}\right) ; 4.05(\mathrm{H}-3) ; 2.89(\mathrm{H}-11)$; 2.67 (H-4); 1.51 (H-13).

${ }^{13} \mathrm{C}-\mathrm{NMR}(50.3 \mathrm{MHz}) \mathrm{CDCl}_{3} / \mathrm{TMS}(\boldsymbol{\delta}=\mathrm{ppm}) .: 145.38$ (C-6); 145.20 (C-7); 125.79 (C-10); 107.55 (C-5); 105.09 (C-8); $100.02\left(\mathrm{OCH}_{2} \mathrm{O}\right) ; 58.87$ (C-3); $42.02(\mathrm{C}-11) ; 28.57$ (C-4); 27.50 (C-13).

[1-(1-Methyl-6,7-methylenedioxy)-isochromanyl]-acetyl arylhydrazone of p-methoxybenzaldehyde $(3 f-(E),(Z))$

m.p.: $168-169^{\circ} \mathrm{C}$

I.R. $(\mathrm{KBr})$ v: 3452, $3170(\mathrm{NH}) ; 1661(\mathrm{C}=\mathrm{O}) \mathrm{cm}^{-1}$.

(E) Isomer

${ }^{1} \mathrm{H}-\mathrm{NMR}(200 \mathrm{MHz}) \mathrm{CDCl}_{3} / \mathrm{TMS}(\delta=\mathrm{ppm}) .: 9.75$ (s,NH); 7.92 (s,H-7'); 7.85 (m,H-3' 5'); 7.56 (m,H-2'6'); $6.58(\mathrm{~s}, \mathrm{H}-8) ; 6.48(\mathrm{~s}, \mathrm{H}-5) ; 5.87\left(\mathrm{~s}, \mathrm{OCH}_{2} \mathrm{O}\right) ; 4.02(\mathrm{~m}, 2 \mathrm{H}-3)$; $3.79\left(\mathrm{~s}, \mathrm{OCH}_{3}\right) ; 2.9(\mathrm{~d}, \mathrm{~J}=15,2 \mathrm{H}-11) ; 2.8(\mathrm{~d}, \mathrm{~J}=15,2 \mathrm{H}-11)$; 2.7 (m,2H-3); 1.55 (3H-13).

${ }^{13} \mathrm{C}-\mathrm{NMR}(50.3 \mathrm{MHz}) \mathrm{CDCl}_{3} / \mathrm{TMS}(\delta=\mathrm{ppm}) .: 166.20$ $(\mathrm{C}=\mathrm{O}) ; 161.23$ (C-4'); 147.06 (C-7'); 146.49 (C-6); 146.42 (C-7); 132.55 (C-9); 129.05 (C-2'6'); 126.67 (C-1'); 125.85 (C-10); 113.90 (C-3'5'); 108.26 (C-5); 105.26 (C8); $100.79\left(\mathrm{OCH}_{2} \mathrm{O}\right) ; 59.72(\mathrm{C}-3) ; 55.16\left(\mathrm{OCH}_{3}\right) ; 48.43$ (C-11); 29.22 (C-4); 27.20 (C-13).

(Z) Isomer

${ }^{1} \mathrm{H}-\mathrm{NMR}(200 \mathrm{MHz}) \mathrm{CDCl}_{3} / \mathrm{TMS}(\delta=\mathrm{ppm}) .: 9.34$ (s,NH); 7.85 (m, H-3' 5'); 7.65 (s,H-7'); 7.56 (m,H-2' 6'); 6.65 (s,H-8); 6.49 (s, H-5); $5.87\left(\mathrm{~s}, \mathrm{OCH}_{2} \mathrm{O}\right) ; 4.02(\mathrm{~m}$, $2 \mathrm{H}-3) ; 3.45(\mathrm{~d}, \mathrm{~J}=13,2 \mathrm{H}-11) ; 3.07(\mathrm{~d}, \mathrm{~J}=13,2 \mathrm{H}-11) ; 2.7$ (m, 2H-3); 1.62 (s, 3H-13).

${ }^{13} \mathrm{C}-\mathrm{NMR}(50.3 \mathrm{MHz}) \mathrm{CDCl}_{3} / \mathrm{TMS}(\boldsymbol{\delta}=\mathrm{ppm}: 172.39$ $(\mathrm{C}=\mathrm{O}) ; 160.94$ (C-4'); 145.92 (C-6); 145.78 (C-7); 143.32 (C-7'); 134.77 (C-9); 128.42 (C-2'6'); 126.52 (C-1'); 126.30 (C-10); 114.05 (C-3'5'); 108.26 (C-5), 105.42 (C-
8); $100.59\left(\mathrm{OCH}_{2} \mathrm{O}\right) ; 59.86(\mathrm{C}-3) ; 55.16\left(\mathrm{OCH}_{3}\right) ; 42.60$ (C-11); 29.22 (C-4); 28.40 (C-13).

[1-(1-Methyl-6,7-methylenedioxy)-isochromanyl]-acetyl arylhydrazone of p-bromobenzaldehyde $(3 g-(E),(Z))$

m.p.: $178-181^{\circ} \mathrm{C}$

máx. (KBr) v: 3450, $3180(\mathrm{NH}) ; 1662(\mathrm{C}=\mathrm{O}) \mathrm{cm}^{-1}$.

(E) Isomer

${ }^{1} \mathrm{H}-\mathrm{NMR}(200 \mathrm{MHz}) \mathrm{CDCl}_{3} / \mathrm{TMS}(\delta=\mathrm{ppm}) .: 9.85$ (s,NH); 7.99 (s,H-7'); 7.7-7.2 (H-2'6'); 7.7-7.2 (H-3'5'); 6.58 (s,H-8); 6.49 (s,H-5); $5.89\left(\mathrm{~s}, \mathrm{OCH}_{2} \mathrm{O}\right) ; 4.03(2 \mathrm{H}-3)$; $2.85(\mathrm{~d}, \mathrm{~J}=15,2 \mathrm{H}-11) ; 2.82(\mathrm{~d}, \mathrm{~J}=15,2 \mathrm{H}-11) ; 2.67(2 \mathrm{H}-4)$; 1.62 (s, 3H-13).

${ }^{13} \mathrm{C}-\mathrm{NMR}(50.3 \mathrm{MHz}) \mathrm{CDCl}_{3} / \mathrm{TMS}(\delta=\mathrm{ppm}) .: 166.53$ $(\mathrm{C}=\mathrm{O}) ; 146.46$ (C-6); 146.35 (C-7); 145.88 (C-7'); 132.85 (C-9); 132.22 (C-1'); 131.66 (C-3'5'); 128.82 (C-2'6'); 125.78 (C-10); 124.38 (C-4'); 108.30 (C-5); 105.38 (C-8); $100.87\left(\mathrm{OCH}_{2} \mathrm{O}\right) ; 59.78(\mathrm{C}-3) ; 48.51(\mathrm{C}-11) ; 29.22(\mathrm{C}-4)$; $27.21(\mathrm{C}-13)$.

(Z) Isomer

${ }^{1} \mathrm{H}-\mathrm{NMR}(200 \mathrm{MHz}) \mathrm{CDCl}_{3} / \mathrm{TMS}(\delta=\mathrm{ppm}) .: 9.67$ (s,NH); 7.67 (s,H-7'); 7.7-7.2 (H-2'6'); 7.7-7.2 (H-3' ''); 6.63 (H-8); 6.51 (s); $5.83\left(\mathrm{OCH}_{2} \mathrm{O}\right) ; 3.84(\mathrm{~m}, 2 \mathrm{H}-3) ; 3.48$ $(\mathrm{d}, \mathrm{J}=13,2 \mathrm{H}-11) ; 3.11(\mathrm{~d}, \mathrm{~J}=13,2 \mathrm{H}-11) ; 2.67$ (m, 2H-4); 1.71 (s, 3H-13).

${ }^{13} \mathrm{C}-\mathrm{NMR}(50.3 \mathrm{MHz}) \mathrm{CDCl}_{3} / \mathrm{TMS}(\delta=\mathrm{ppm}) .: 172.89$ $(\mathrm{C}=\mathrm{O}) ; 146.46$ (C-6); 146.35 (C-7); 142.43 (C-7'); 134.50 (C-9); 132.52 (C-1'); 131.80 (C-3'5'); 128.30 (C-2'6'); 126.52 (C-10); 123.93 (C-4'); 108.30 (C-5); 105.50 (C-8); $100.68\left(\mathrm{OCH}_{2} \mathrm{O}\right) ; 75.90(\mathrm{C}-1) ; 59.91(\mathrm{C}-3) ; 42.61$ (C-11); 29.22 (C-4); 28.41 (C-13).

\section{Pharmacology}

The analgesic activity was determined in vivo using the abdominal constriction test induced by $0.6 \%$ acetic acid $(0.1 \mathrm{~mL} / 10 \mathrm{~g})$ in albino $\mathrm{mice}^{24}(c a .20 \mathrm{~g})$; indomethacin was used as the standard. The solution of acetic acid was administered i.p. and the number of constrictions was registered for $30 \mathrm{~min}$ (control). All compounds were administered p.o. in arabic gum (5\%) in a dose of $100 \mathrm{mM} / \mathrm{kg}(0.1 \mathrm{~mL} / 20 \mathrm{~g})$ $1 \mathrm{~h}$ before the injection of the acetic acid solution. The data were statistically analyzed by the Student t-test, with the significance level set at $P<0.05$.

\section{Results and Discussion}

The synthesis of new compounds (3a-g) was carried out by exploring the previously described ester $(5)^{16}$ synthesized from natural safrole (4) in $80 \%$ yield. This synthetic route uses as key step an acid-catalyzed intramolecular alkylation of the 6-position of the aromatic system of the hemiketal intermediate (7), formed in situ from the condensation of homopiperonilic alcohol (8) with ethylacetoacetate (9), in the presence of the catalytic amount of 


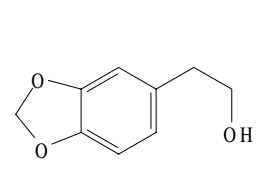

(8)

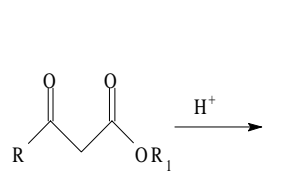

(9)

$$
\begin{aligned}
& \mathrm{R}=\mathrm{CH}_{3} \\
& \mathrm{R}_{1}=\mathrm{C}_{2} \mathrm{H}_{5}
\end{aligned}
$$

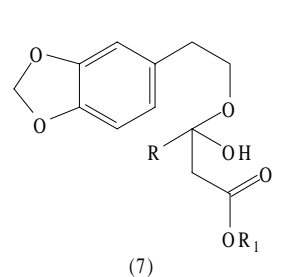

(7)

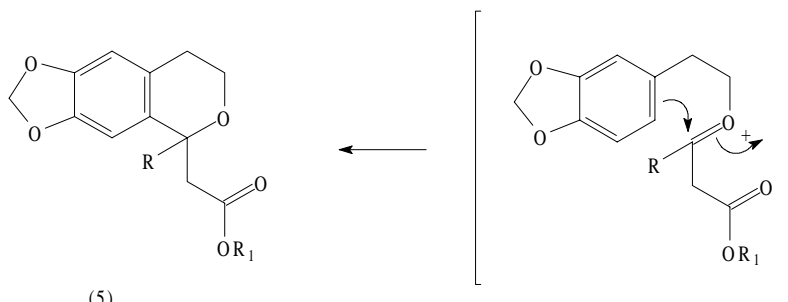

Scheme 2.

(5)

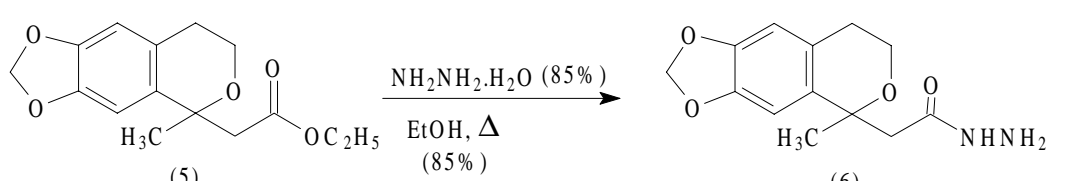

(5) $(85 \%)$

(6)

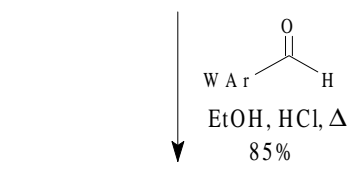

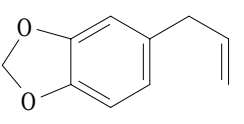

(4)

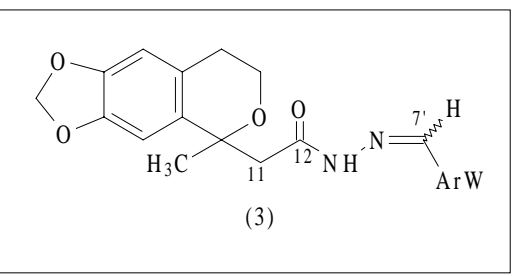

Scheme 3 .

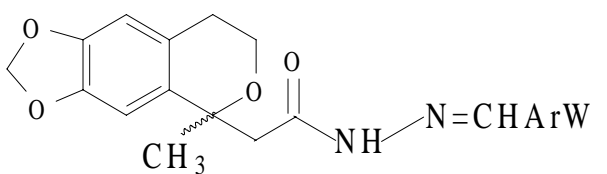

Figure 1. Possible tautomeric forms of compound 3.

p-toluenesulfonic acid, as indicated in Scheme 2. The isochromanylacylhydrazide (6), precursor of the desired derivatives (3), was prepared in $85 \%$ yield by the conventional treatment of an ethanolic solution of 1-alkyl1-isochroman ester (5) with $85 \%$ hydrazine hydrate, at reflux, to furnish (6) in $85 \%$ yield, as outlined in Scheme 3 . Finally, the target compounds (3a-g) were prepared in high yield by acidic condensation of (6) with the appropriate aldehydes ${ }^{20}$ (Table 1 ). The pattern and choice of the substituent (Ar-W), present in the acylarylhydrazone derivatives ( $3 \mathrm{a}-\mathrm{g}$ ), was determined by the need to introduce a significant difference in the electronic character in this framework $^{21}$, in order to study the eventual contribution of these structural modifications to bioactivity. Apart from this, the presence of an N-dimethylaminophenyl unit present in (3b) was indicated by the previously described potent analgesic activity detected in compound $(2)^{11}$. 
These new derivatives (3a-g) were fully characterized spectroscopically. For instance, in the infrared spectra the presence of a strong absorption at $v 3450 \mathrm{~cm}^{-1}$ is attributed to an -NH group, indicating that the acylhydrazone form predominates over the azo form (Fig. 1) ${ }^{22}$. Careful analysis of hydrogen and carbon-13 nuclear magnetic resonance (NMR) spectra of these derivatives (3a-g) indicated the presence of a diastereomeric mixture at the $\mathrm{C}=\mathrm{N}$ double bond level, in a 70:30 ratio favoring the $(E)$-isomer. The analysis of the homonuclear $\left({ }^{1} \mathrm{Hx}{ }^{1} \mathrm{H}-\mathrm{COSY}\right)$ and heteronuclear $\left[{ }^{1} \mathrm{Hx}{ }^{13} \mathrm{C}\right.$-COSY:modulated with ${ }^{1} \mathrm{~J}_{\mathrm{CH}}$ and ${ }^{\mathrm{n}} \mathrm{J}_{\mathrm{CH}}(\mathrm{n}=2$ and 3,COLOC)] 2D shift-correlated spectra confirmed this assumption, especially by the pattern presented by the $\mathrm{H}-7^{\prime}$, C-7', $\mathrm{CH}_{2}-11, \mathrm{C}-12$ and $\mathrm{NH}-12$, indicating that the (Z)-isomer resonates at a higher magnetic field than the $(E)$-isomer (Table 2).

These new isochromanylarylacetylhydrazone derivatives (3a-g) were evaluated for their analgesic and anti-inflammatory activities. Unfortunately, these compounds were not effective as anti-inflammatory agents, as observed in the carrageenan-induced rat paw edema test ${ }^{23}$. In this bioassay, only compound $3 \mathrm{~g}$, where $\mathrm{W}=\mathrm{Br}$, presented

Table 2. ${ }^{1} \mathrm{H}$ and ${ }^{13} \mathrm{C}$ nuclear magnetic resonance data of compound $3 \mathrm{a}$.

\begin{tabular}{|c|c|c|c|c|}
\hline \multicolumn{5}{|c|}{${ }^{1} \mathrm{H} x{ }^{13} \mathrm{C}-\mathrm{COSY}-{ }^{1} \mathrm{JCH}_{\mathrm{CH}}$} \\
\hline & \multicolumn{2}{|c|}{$3 \mathbf{a}(E)$} & \multicolumn{2}{|c|}{$\mathbf{3 a}(Z)$} \\
\hline & $\delta_{\mathrm{C}}$ & $\delta_{\mathrm{H}}$ & $\delta_{\mathrm{C}}$ & $\delta_{\mathrm{H}}$ \\
\hline $7^{\prime}$ & 147,80 & 8,0 & 143,70 & 7,7 \\
\hline 11 & 48,37 & 3,0 & 42,50 & 3,4 \\
\hline 12 & 166,40 & & 172,87 & \\
\hline
\end{tabular}

significant activity, inhibiting paw edema in $30.9 \%$ in a dose of $100 \mu \mathrm{mol} / \mathrm{kg}$ p.o. The anti-inflammatory test results indicated that the enhanced lipophilicity of the $p$-bromophenyl template may contribute to the observed activity. The analgesic activity of these compounds was investigated (Table 3) by the inductive abdominal constrictions test in mice ${ }^{24}$, using indomethacin at $30 \mu \mathrm{mol} / \mathrm{kg}$, p.o., as a standard. The compound possessing the 4-dimethylaminophenyl moiety at the acylhydrazone framework (3c), was the most potent one. In fact, this result seems to indicate, as observed in an other series previously synthesized in our laboratory ${ }^{25}$, that the presence of this functionality could have an important role in analgesic activity (Fig. 2), probably acting as a hydrogen bond donor in the interaction with the bioreceptor.

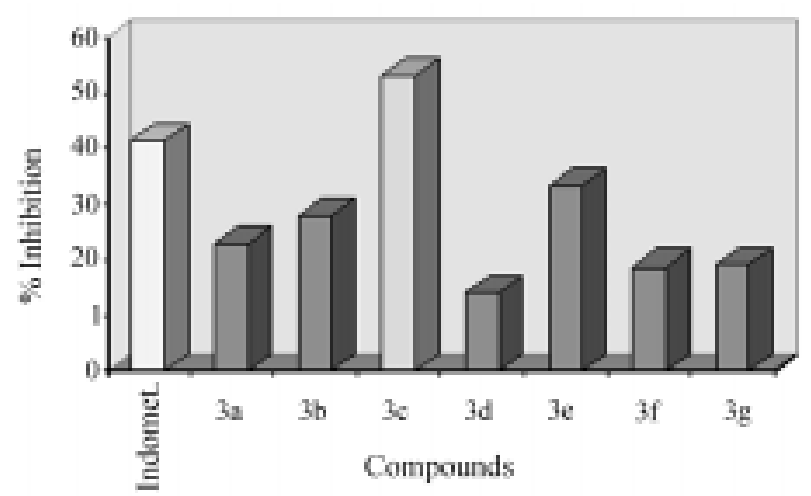

Figure 2. The in vivo effect of isochromanyacetylarylhydrazone derivatives (3a-3g) and indomethacin on the abdominal constrictions induced by acetic acid in mice.

Table 3. The in vivo effect of isochromanylacetylarylhydrazone derivatives (3a-3g) (100 $\mu \mathrm{M} / \mathrm{kg}$, p.o.) and indomethacin $(30 \mu \mathrm{M} / \mathrm{kg}$, p.o.) in the test of mouse abdominal constrictions induced by acetic acid solution $(0.6 \%$, i.p.).

\begin{tabular}{|c|c|c|c|c|}
\hline Compound $^{\mathrm{a}}$ & Concentration $\mu$ moles $/ \mathrm{kg}$ & $\mathrm{N}$ & $\mathrm{N}^{\circ}$ Constrictions $^{\mathrm{b}}$ & Inhibition\% \\
\hline Control & & 35 & $99.3 \pm 33.5$ & - \\
\hline Vehicle & - & 30 & $91.8 \pm 4.2$ & 7.5 \\
\hline Indomethacin $^{c}$ & 30 & 8 & $43.4 \pm 7.6$ & $41.4^{*}$ \\
\hline $3 a$ & 100 & 10 & $76.5 \pm 3.3$ & 22.9 n.s. \\
\hline $3 b$ & 100 & 10 & $71.7 \pm 3.0$ & 27.8 n.s. \\
\hline $3 c$ & 100 & 9 & $46.4 \pm 7.2$ & $53.3^{*}$ \\
\hline $3 d$ & 100 & 7 & $85.3 \pm 7.8$ & 14.1 n.s. \\
\hline $3 e$ & 100 & 11 & $66.2 \pm 8.0$ & $33.3^{*}$ \\
\hline $3 f$ & 100 & 7 & $80.7 \pm 7.8$ & 18.7 n.s. \\
\hline $3 g$ & 100 & 5 & $80.2 \pm 4.8$ & 19.2 n.s. \\
\hline
\end{tabular}

$\mathrm{N}=$ number of animals; n.s. = not significant; *p < 0.05 (Student "t" test); a) All compounds (3a-3g) were administered in arabic gum (5\%), p.o., in a dose of $100 \mu \mathrm{M} / \mathrm{kg}(0.1 \mathrm{~mL} / 20 \mathrm{~g}) 1 \mathrm{~h}$ before injection of the acetic acid solution; b) The abdominal constrictions test induced by acetic acid $0.6 \%(0,1$ $\mathrm{mL} / 10 \mathrm{~g})$, i.p., in albino mice; c) indomethacin was used as the standard, administered in arabic gum (5\%), p.o., in a dose of $30 \mu \mathrm{M} / \mathrm{kg}(0.1 \mathrm{~mL} / 20 \mathrm{~g})$ $1 \mathrm{~h}$ before the injection of the acetic acid solution. 


\section{Conclusions}

The synthetic methodology used in this work was shown to be efficient for obtaining of new isochromanylacetylhydrazone compounds (3a-g) in high yields, using natural safrole as the starting material. These compounds represent a new class of non-addictive analgesic agents.

The hydrogen and carbon-13 nuclear magnetic resonance (NMR) spectra and assignments by the homonuclear $\left({ }^{1} \mathrm{Hx}^{1} \mathrm{H}-\right.$ $\mathrm{COSY}$ ) and heteronuclear $\left[{ }^{1} \mathrm{Hx}{ }^{13} \mathrm{C}-\mathrm{COSY}\right.$ :modulated with ${ }^{1} \mathrm{~J}_{\mathrm{CH}}$ and ${ }^{\mathrm{n}} \mathrm{J}_{\mathrm{CH}}(\mathrm{n}=2$ and 3, COLOC)] 2D shift-correlated spectra showed that the $(E)$ diastereoisomer makes a major contribution in the desired compound (3).

The results described reinforce, as previously observed in an other related series of acylhydrazones synthesized in our laboratory ${ }^{25}$, that the acylhydrazone moiety is an important pharmacophore group for the observed activity, and that the $\mathrm{N}$-dimethyl substituent at position 4 of the phenyl ring is essential for analgesic activity. The mechanism of the action of these new compounds is currently under investigation in our laboratory and will be disclosed in the future.

\section{Acknowledgments}

We are grateful to CNPq (\#52.0511/94-3), FAPERJ (E120-33/5), and FUJB-UFRJ (BR) for the financial support for this work, and to CNPq and CAPES (BR) for fellowships ( MRLS, ALPM, and EJB). The authors thank Prof. Mario Geraldo (UFRRJ-BR) for the NMR spectra, the referees for the valuable suggestions, and S. de C. Parrini, K.C.M. da Silva, G. Barreiro, and Dr. A.L.P. de Miranda (LASSBio, UFRJ-BR) for the biological data.

\section{References}

1. Handwerker, H.O. Sensory Functions of Skin; Pergamon Press, Oxford ,1976; p. 427.

2. Ferreira, S.H.; Moncada, S.; Vane, J.R. Br. J. Pharmacol. 1973, 49, 86.

3. Schepelmann, K.; Meßllinger, K.; Schaible, H-G.; Schimdt, R.F. Neuroscience 1992, 50, 237.

4. Meade, E.A.; Smith, W.L.; De Witt, D.L. J. Biol. Chem. 1993, 268, 6610.

5. Kujubu, D.A.; Fletcher, B.B.S.; Varnum, B.C.; Lim, R.W.; Herschman, H.R. J. Biol. Chem. 1991, 266,12866.

6. Patrignani, P.; Panara, M.R.; Greco, A.; Fusio, O.; Natoli, C.; Iacobelli, S.; Cipollone, F.; Ganci, A.; Créminon, C.; Maclouf, J.; Patrono, C. J. Pharmacol. Exp. Ther. 1994, 271, 1705.
7. Flower, R.J.; Vane, J.R. Biochem. Pharmacol. 1974, 23, 1439 .

8. Bray, M. A. Agents Action 1986, 19, 87.

9. Brain, S.D.; Williams, T.J. Pharmac. Ther. 1990, 46, 57.

10. Barreiro, E.J.; Costa, P.R.R.; Barros, P.R.V.R.; de Mello, R.T. An. Acad. Bras. Cienc. 1981, 53, 65.

11. Matheus, M.E.; Oliveira, L.F.; Freitas, A.C.C.; Carvalho, A.M.A.S.P.; Barreiro, E.J. Braz. J. Med. Biol. Res. 1991, 24, 1219.

12. Silveira, I.A.B.F.; Paulo, L.G.; Miranda, A.L.P.; Rocha, S.O.; Freitas, A. C. C.; Barreiro, E.J. J. Pharm. Pharmacol. 1993, 45, 646.

13. Fraga, C.A.M.; Barreiro, E.J. J. Heterocyclic Chem. 1992, 29, 1667.

14. Freitas, A.C.C.; Barreiro, E.J. J. Heterocyclic Chem. 1992, 29,407.

15. Lima, M.E.F.; Barreiro, E.J. J. Pharm. Sciences 1992, $81,1219$.

16. Silva, E.F.; Barreiro, E.J. J. Braz. Chem. Soc. 1993, 4, 40.

17. Dias, L.R.S.; Alvim, M.J.F.; Freitas, A.C.C.; Barreiro, E.J., Miranda, A.L.P. Pharmac. Acta Helvetiae 1994, 69, 163.

18. Freitas, A.C.C. PhD Thesis, Instituto de Química/ LASSBio, Universidade Federal do Rio de Janeiro, 1991.

19. Barreiro, E.J.; Costa, P.R.R.; Coelho, F.A.S.; Farias, F.M.C. J. Chem. Res. (M) 1985, 2301.

20. Santos, M.R.L.; Barreiro, E.J.; Braz-Filho, R. Abstracts of $18^{a}$ Reunião Anual da Sociedade Brasileira de Química, Braz. Chem. Soc., Caxambu, Brazil, 1995, Abstr. QO-074.

21. Kubiniy, H. Hansch Analysis and Related Approaches; Weinheim, Germany, 1993, p 23.

22. Szileigye, G.; Kaszheimer, E.; Matyus, P.; Kosary, J.; Czarc, K.; Czen, G.; Huszti, Z.; Tardos, L.; Kcsa, E.; Jaszlits, L. Eur. J. Med. Chem. 1984, 19, 111.

23. Ferreira, S.H. J. Pharm. Pharmacol. 1979, 31, 648.

24. Whittle, B.A. Br. J. Pharmacol. 1964, 22, 246.

25. Miranda, A.L.P.; Santos, M.R.L.; Lima, P.; Barreiro, E.J.; Braz-Filho, R.; Silva, K.C.M.; Parrini, S.C.; Abstracts of X Reunião Anual da Federação de Sociedade de Biologia Experimental, Caxambu, Brazil, 1995, Abstr. 19.006. 